\title{
Minimally invasive esophagectomy learning curves with different types of background experience
}

\author{
Olli Helminen $^{1,2} \wedge$, Joonas H. Kauppila ${ }^{1,3}$, Henna Saviaro ${ }^{1}$, Fredrik Yannopoulos ${ }^{1,4}$, Sanna Meriläinen $^{1}$, \\ Vesa Koivukangas ${ }^{1}$, Heikki Huhta ${ }^{1}$, Johanna Mrena ${ }^{2}$, Juha Saarnio ${ }^{1}$, Eero Sihvo ${ }^{2}$ \\ ${ }^{1}$ Surgery Research Unit, Medical Research Center Oulu, Oulu University Hospital, University of Oulu, Oulu, Finland; ${ }^{2}$ Department of Surgery, \\ Central Finland Central Hospital, Jyväskylä, Finland; ${ }^{3}$ Upper Gastrointestinal Surgery, Department of Molecular Medicine and Surgery, Karolinska \\ Institute, Karolinska University Hospital, Stockholm, Sweden; ${ }^{4}$ Department of Cardiothoracic Surgery, Oulu University Hospital, Oulu, Finland \\ Contributions: (I) Conception and design: O Helminen, JH Kauppila, H Huhta, E Sihvo; (II) Administrative support: All authors; (III) Provision of \\ study materials or patients: All authors; (IV) Collection and assembly of data: All authors; (V) Data analysis and interpretation: O Helminen, JH \\ Kauppila, F Yannopoulos, E Sihvo; (VI) Manuscript writing: All authors; (VII) Final approval of manuscript: All authors. \\ Correspondence to: Olli Helminen, MD, PhD. Department of Surgery, Oulu University Hospital, Kajaanintie 50, 90220 Oulu, Finland. \\ Email: olli.helminen@oulu.fi.
}

Background: Minimally invasive esophagectomy (MIE) is a complex procedure with learning associated morbidity. The aim was to evaluate the learning curve for MIE focusing on short-term outcomes in two settings: (I) experienced MIE surgeon in new hospital (Hospital 1); (II) surgeons experienced with open esophagectomy and minimally invasive surrogate surgery (Hospital 2).

Methods: In Hospital 1 and Hospital 2, on intent-to-treat basis number of MIEs were 132 and 57 , respectively. The primary outcomes were major complications and anastomosis leaks. Secondary outcomes were operative time, blood loss, lymph node yield, hospital stay and 1-year mortality. Length of learning curves were analyzed with risk-adjusted cumulative sum (RA-CUSUM) method.

Results: In Hospital 1, major complication and anastomosis leak rates were $9.8 \%$ and $4.5 \%, 22.8 \%$ and $12.3 \%$ in Hospital 2, respectively. In Hospital 1, complication and leak rates remained stable. In Hospital 2, improvement occurred after 34 cases in major complications and 29 cases in leaks. Of secondary outcomes, improvements were seen in Hospital 1 in operative time after 61, blood loss after 86, lymph node yield after 52, hospital stay after 19 and 1-year mortality after 24 cases. In Hospital 2, improvement occurred in operative time after 30 , blood loss after 15, lymph node yield after 45, hospital stay after 50 and 1-year mortality after 15 cases.

Conclusions: According to this study, learning phase of the individual surgeon determines the outcomes of MIE, not the institutional learning phase.

Keywords: Minimally invasive esophagectomy (MIE); learning curve; cumulative sum (CUSUM); surrogate surgery; esophageal cancer

Submitted Jun 25, 2021. Accepted for publication Sep 23, 2021.

doi: $10.21037 /$ jtd-21-1063

View this article at: https://dx.doi.org/10.21037/jtd-21-1063

^ ORCID: 0000-0001-9544-6475. 


\section{Introduction}

Esophageal cancer is the sixth leading cause of cancerrelated mortality (1). Five-year survival rates have improved up to $56.5 \%$ in a population-based setting (2). Esophagectomy is characterized by frequent complications and 90 -day mortality of $6.4 \%$ (3).

To reduce surgery-associated morbidity, minimally invasive esophagectomy (MIE) has been increasingly used. MIE is a complex procedure and associated with learningrelated extra morbidity such as anastomotic leaks (4). This is especially true with Ivor Lewis MIE (5).

Due to heterogeneous methodologies in learning curve studies, it is challenging to draw any general conclusions. Generally, the larger the study, the longer the learning curve (6). With such variable outcome parameters as operative time, blood loss, lymph node yield, hospital stay and postoperative complication, learning curve for MIE has varied from 20 to 175 cases (6).

The aim of this study was to evaluate the learning curve for MIE with variable outcome parameters in two different settings. First, an experienced MIE surgeon implemented a new MIE program in a center not previously performing MIE. Second, surgeons experienced with open esophagectomy and minimally invasive surrogate surgery implemented a new MIE program at their center.

We present the following article in accordance with the STROBE reporting checklist (available at https://dx.doi. org/10.21037/jtd-21-1063).

\section{Methods}

\section{Study design}

The study was conducted according to the guidelines of the Declaration of Helsinki (as revised in 2013). The study was approved by the Oulu University Hospital Ethics Committee and the hospital districts (committee's reference number 81/2008). The need to obtain informed consent from the study patients was waived by the Finnish National Authority for Medicolegal Affairs (VALVIRA).

All patients who underwent intention-to-treat MIE for esophageal or gastroesophageal junction cancer in Central Finland Central Hospital (Hospital 1) and Oulu University Hospital (Hospital 2) were eligible for this retrospective cohort study. Benign diseases and operations aborted due to inoperable disease, i.e., occult metastases or carcinosis, were excluded.

The clinical data from prospectively kept records was re- reviewed retrospectively by the investigators. Complications were graded according to Clavien-Dindo classification (7). Major complication was defined as Clavien-Dindo $\geq 3$ a. Definitions for complications according to the Esophagectomy Complications Consensus Group (ECCG) were strictly followed (8). Results were compared to previously presented Benchmark values (9). Length of the learning curve was calculated using the Risk-adjusted cumulative sum (RA-CUSUM) method (10). This study was approved by the hospital districts.

The primary outcomes were major complication and anastomotic leak rate [diagnosed with either endoscopy or computed tomography (CT) with oral contrast]. Secondary outcomes included operative time, blood loss, lymph node yield, radicality (R0/1/2), conversion rate, hospital stay, readmissions, 90-day mortality and 1-year mortality.

\section{Patients and hospitals}

In Hospital 1, MIE program started in September 2012 by a surgeon who had done his first self-learned hybrid esophagectomy in 2007. In 2009, after his colleague finished MIE fellowship, they started formal MIE program in Helsinki University Hospital. After performing 49 MIEs or hybrid procedures and more than 100 open esophagectomies together with a significant experience of minimally invasive lung cancer surgery, new program was started (11). In Hospital 2, MIE program was started in September 2017 by two experienced upper GI-surgeons with high surrogate surgery volume, including laparoscopic gastrectomy, hiatal hernia and bariatric surgery. Team included a thoracic surgeon with expertise in thoracoscopic surgery. Before first MIE, multiple visits in expert clinics were executed. By August 2021, total MIE volume for cancer in Hospital 1 was 132 and in Hospital 2 57. Followup ended August 31, 2021.

\section{Operative approach}

The preoperative protocol included endoscopy with biopsies, endoscopic ultrasound, CT, and positron emission tomography (PET) CT. Endoscopic ultrasound was performed in selected cases to assess the possibility of endoscopic therapy, or the need for neoadjuvant treatment for endoscopically small, superficial tumors. PET-CT was performed routinely in Hospital 1, and selectively in Hospital 2. Diagnostic laparoscopy was performed selectively to exclude peritoneal metastases or 
to supplement inconclusive radiological staging. According to the current ESMO guidelines (12), patients with clinical T3 and/or N+ tumors were referred to neoadjuvant/ perioperative treatment. Neoadjuvant treatment regimen has been previously described in detail (11). Patients receiving neoadjuvant treatment were restaged before the surgery with CT or PET-CT according to primary fluorodeoxyglucose avidity. The operation was performed usually after a 6-week recovery period.

A minimally invasive Ivor Lewis esophagectomy with en-bloc lymphadenectomy and intrathoracic anastomosis was the preferred approach in both Hospital $1(n=116$, 87.9\%) (11) and Hospital 2 ( $\mathrm{n}=55,96.5 \%)$. The rest of the operations were McKeown type MIEs. In Hospital 1 , intrathoracic end-to-side anastomosis was completed after purse string suture around the cap using a circular stapler and was reinforced with an omental flap. Side-toside anastomosis with a linear stapler, during early learning period ( $\mathrm{n}=11)$ in Hospital 2, was performed. Thereafter, the technique was switched to end-to-side anastomoses with EEA stapler with purse-string suture around perorally placed OrVil device. In Hospital 1, 2D optics was changed to $3 \mathrm{D}$ from case 21 and to better quality $3 \mathrm{D}$ from case 80. In Hospital 2, 3D was used throughout the study. In Hospital 1, ICG-guided tailored lymphadenectomy series was performed from case 57 to 63 (13). Surgery included a selective feeding jejunostomy and endoscopic pyloric dilatation in all patients.

Perioperative care included the assessment and optimization of medical risk factors, thromboprophylaxis with low-molecular-weight heparin and intermittent pneumatic compression stockings, prophylactic antibiotics, standardized anaesthesia with epidural analgesia, avoidance of hypothermia, and early mobilization.

All but 2 patients in Hospital 1 and 3 in Hospital 2 completed a minimum of 90-day follow-up. In Hospital 1, 123/132 and in Hospital 2, 38/57 patients reached 1-year follow-up or had died at the end of current study. Mortality data was confirmed from the nationwide compulsory Cause of Death registry held by Statistics Finland.

\section{Statistical analysis}

The RA-CUSUM curves for anastomotic leaks (yes/no), major complications (yes/no), operation time, blood loss, lymph node yield, hospital stay and 1-year mortality (yes/no operated before August 31, 2020) were formed using R version 4.0.3 and Microsoft Excel for Mac version 15.29.1 (Redmond, WA, USA). The outcomes without established cutoffs (operation time, blood loss, lymph node yield, and hospital stay) were stratified by the center median. First, predicted risks of each outcome were calculated using the total dataset using multivariable logistic regression with prespecified variables. Risk scores for age ( $<75$ years $>75$ years), sex (male/female), histology (adenocarcinoma/squamous cell carcinoma/other), tumor stage (0-I/II/III/IV) and neoadjuvant therapy (yes/no). No or very few outcomes occurred in some logistic regression models including stage IV or female patients as separate categories. As the real effects of these variables on outcome risk were clinically assumed to be minor, tumor stage was grouped 0-I, II, or III-IV in analyses for anastomotic leak, major complications, length of stay, and 1-year mortality, and sex was excluded from the model for anastomotic leak to avoid nonconvergence of logistic regression. Individual risks for outcomes were used to assess expected outcome in RACUSUM. The curves depict cumulative difference between the observed and expected mortality/survival on the $y$-axis, equated as $\mathrm{Si}=\mathrm{Si}-1+(\Sigma \mathrm{i}-\Sigma \mathrm{R}) ; \mathrm{S} 0=0$. In the equation, $\mathrm{Si}$ is the cumulative sum, $\Sigma i$ is the sum of events at procedure number $\mathrm{i}$, and $\Sigma \mathrm{R}$ is the sum of expected events at procedure number i. In the RA-CUSUM equation, observations exceeding the expected risk of the outcome increase, and observations is below the expected risk decrease CUSUM.

The RA-CUSUMs were expected to increase in the learning phase before a plateau. After plateau, the events occurred less often than expected, and the curve descended. A stable situation showed no clear pattern. Learning was interpreted to be complete at the end of the plateau phase, where a clear learning-plateau-improved performancepattern was observed.

For patients who received neoadjuvant treatment, and had a complete response, clinical stage was used in the statistical analysis instead of the pathological stage. Proportions, means and median values of other measured variables were compared using the chi-squared test and Mann-Whitney U-test as appropriate. Survival times were calculated from the date of surgery until the time of death or the end of follow-up. Kaplan-Meier survival curves were calculated according to the life table method to visualize the crude overall survival. IBM SPSS 26.0 (IBM corp., Armonk, NY, USA) was used. 
Table 1 Baseline characteristics

\begin{tabular}{|c|c|c|c|}
\hline Variable & Hospital 1, n=132 & Hospital 2, n=57 & $P$ value \\
\hline BMI, median [IQR] & 26 [23-28] & 25 [23-29] & 0.790 \\
\hline Sex, male (\%) & $98(74.2)$ & $46(80.7)$ & 0.339 \\
\hline Charlson Comorbidity Index, n (\%) & & & 0.792 \\
\hline 1 & $37(28.0)$ & $15(26.3)$ & \\
\hline$\geq 2$ & $30(22.7)$ & $11(19.3)$ & \\
\hline Histology, n (\%) & & & 0.674 \\
\hline Adenocarcinoma & $106(80.3)$ & $43(75.4)$ & \\
\hline PET-CT, yes & $131(99.2)$ & $27(47.4)$ & $<0.001$ \\
\hline Grade of differentiation, n (\%) & & & 0.617 \\
\hline I & $20(15.2)$ & $9(15.8)$ & \\
\hline II & $58(43.9)$ & $20(35.1)$ & \\
\hline III & $26(19.7)$ & $15(26.3)$ & \\
\hline No data & $28(21.2)$ & $13(22.8)$ & \\
\hline Stage $^{1}, \mathrm{n}(\%)$ & & & 0.150 \\
\hline 0 & $2(1.5)$ & 0 & \\
\hline Upper & 0 & 0 & \\
\hline Middle & $8(6.1)$ & $6(10.5)$ & \\
\hline Lower & $124(93.9)$ & $43(89.5)$ & \\
\hline Neoadjuvant treatment, n (\%) & $102(77.3)$ & $43(75.4)$ & 0.784 \\
\hline
\end{tabular}

\footnotetext{
${ }^{1}$, in patients who received no neoadjuvant treatment, pathological staging was used. In neoadjuvant treated patients with complete
} response, clinical stage was used.

\section{Results}

\section{Patients}

Baseline characteristics are presented in Table 1. Since introduction of MIE program, no open esophagectomy for cancer was performed in Hospital 1, and only two in
Hospital 2. Therefore, the learning curves represent nearly all patients referred to esophagectomy. During the study, the mean annual MIE volume for cancer was 15 in Hospital 1 and 14 in Hospital 2. Majority had locally advanced stage (T3 and/or N1, 87.9\% in Hospital 1 and 93.0\% in Hospital 2). Of all patients, neoadjuvant treatment was given to $77.3 \%$ 
Table 2 Study parameters and Benchmark value

\begin{tabular}{|c|c|c|c|c|}
\hline Variable & Hospital 1, n=132 & Hospital 2, $n=57$ & $P$ value between Hospitals & Benchmark level (9) \\
\hline Neoadjuvant treatment rate ${ }^{1}$ & $102 / 116(87.9 \%)$ & $43 / 52(82.7 \%)$ & 0.361 & \\
\hline \multicolumn{5}{|l|}{ Operation-related } \\
\hline Operative time, median [IQR] & 357 [318-422] & $440[405-495]$ & $<0.001$ & \\
\hline Lymph node yield, median [IQR] & 22 [18-29] & 17 [12-21] & $<0.001$ & $\geq 23$ \\
\hline Radicality (R1 rate) & $2.3 \%$ & $5.3 \%$ & 0.282 & $\leq 3.1 \%$ \\
\hline Conversion & $2.3 \%$ & $3.5 \%$ & 0.627 & \\
\hline \multicolumn{5}{|l|}{ Post-operative } \\
\hline Hospital stay, median [IQR] & 10 [9-12] & $11[9-14]$ & 0.040 & $13[10-21]$ \\
\hline Discharged to home & $85.6 \%$ & $82.5 \%$ & 0.239 & \\
\hline Re-admissions & $9.1 \%$ & $10.5 \%$ & 0.770 & $\leq 18 \%$ \\
\hline 90-day mortality & $1.5 \%$ & $1.8 \%$ & 0.904 & $\leq 4.6 \%$ \\
\hline 1-year mortality & $7.3 \%$ & $18.4 \%$ & 0.046 & $14.5 \%$ \\
\hline
\end{tabular}

in Hospital 1 and 75.4\% in Hospital 2. Guideline-based neoadjuvant treatment rate in patients with locally advanced disease was $87.9 \%$ and $82.7 \%$, respectively (Table 2). PET-CT was performed in $99.2 \%$ and $47.4 \%$, respectively (Table 1). In Hospital 1, case numbers 49, 95, 118 were salvage esophagectomies.

\section{Parameters associated with learning curve}

\section{Operation-related (Figure 1)}

The operative time showed improved performance in Hospital 1 after 61 cases (Figure $1 A$ ) and in Hospital 2 after 30 cases (Figure 1B, Table 2). Change-points in blood loss in RA-CUSUM curves were seen at 86 cases in Hospital 1 (Figure 1C) and at 15 cases in Hospital 2 (Figure 1D). In Hospital 1, lymph node yield increased with a change-point after 52 cases (Figure 1E) and in Hospital 2 after 45 cases (Figure 1F).

Based on the change point in learning curve, median operative time in Hospital 1 of $402 \mathrm{~min}$ (IQR: $355-472 \mathrm{~min}$ ) before case 61 differed significantly $(\mathrm{P}<0.001)$ of that after
327 min (IQR: 289-372 min). Similarly, median blood loss before case 86 was $200 \mathrm{~mL}$ (IQR: 100-300 mL) and after $100 \mathrm{~mL}$ (IQR: $50-170 \mathrm{~mL}$ ), $\mathrm{P}=0.015$ and lymph node yield before case 5220 (IQR: 14-24) and after 26 (IQR: 19-32), $\mathrm{P}<0.001$. In Hospital 2, median operative time until case 30 was $480 \mathrm{~min}$ (IQR: $425-523 \mathrm{~min}$ ) and after $410 \mathrm{~min}$ (IQR: $383-440 \mathrm{~min}$ ), $\mathrm{P}=0.001$. Blood loss until case 15 was $200 \mathrm{~mL}$ (IQR: $200-400 \mathrm{~mL}$ ) and after $100 \mathrm{~mL}$ (IQR: 50-200 mL), $\mathrm{P}=0.039$. Lymph node yield until case 45 was 16 (IQR: 12-20) and after 21 (IQR: 15-33), P=0.088.

\section{Post-operative (Figure 2)}

The major complications and anastomosis leaks are listed in Table 2. In Hospital 1, stable RA-CUSUM was detected in these parameters (Figure 2A,2C). An improvement in Hospital 2 was observed in major complications after 34 cases (Figure 2B) and in anastomosis leaks after 29 cases (Figure 2D).

Median hospital stay in the two Hospitals was 10 (IQR: 9-12) and 11 (IQR: 9-14), respectively (Table 2). Performance improvement in Hospital 1 was seen after 19 cases (Figure 1G), and in Hospital 2 after 50 cases (Figure 1H). 

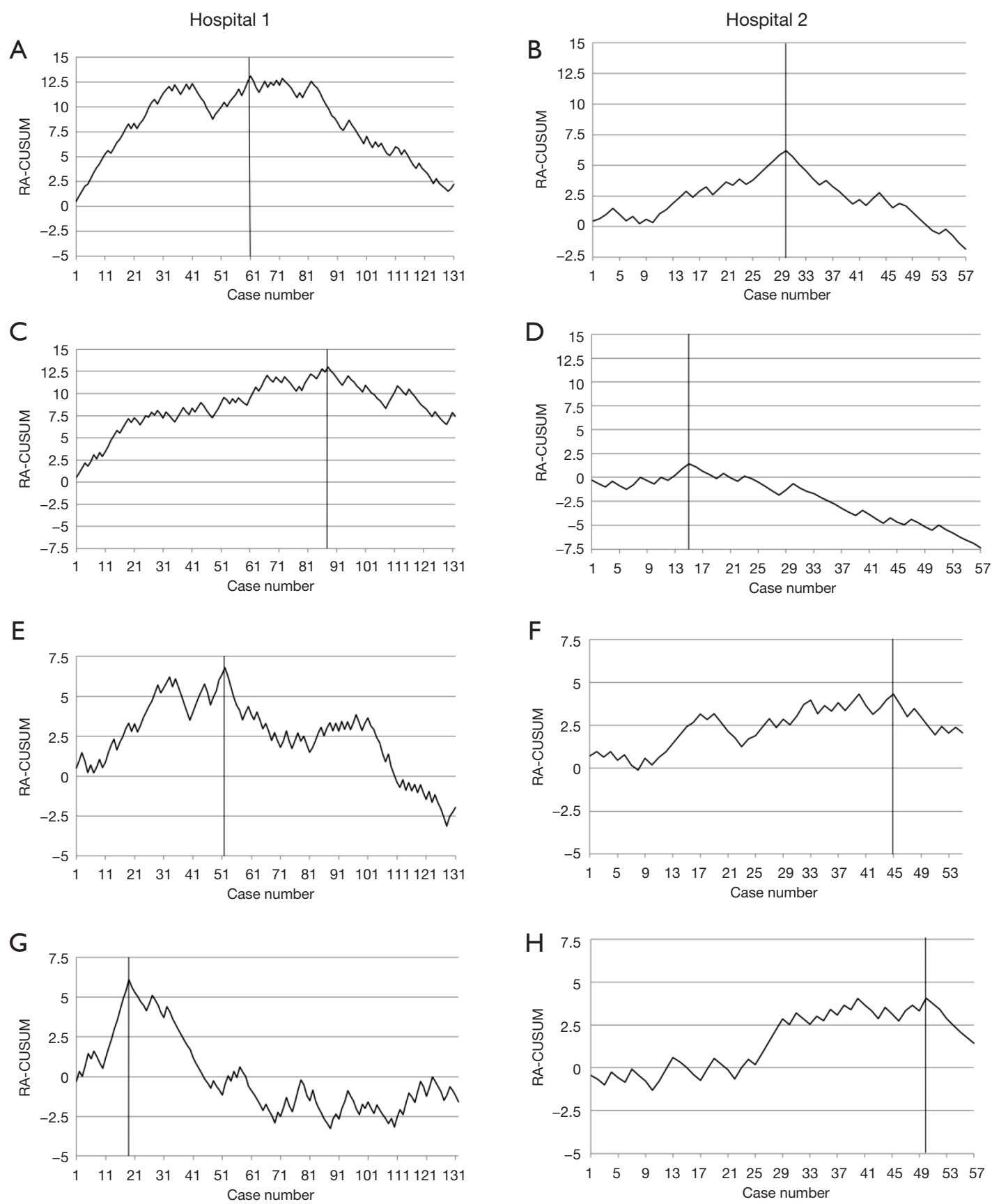

Figure 1 Learning curves presenting intraoperative outcomes and hospital stay. RA-CUSUM of operative time (A,B), blood loss (C,D), lymph node yield (E,F) and hospital stay $(\mathrm{G}, \mathrm{H})$. Hospital 1 is presented in the left, Hospital 2 in the right column. Curve was inverted regarding lymph node yield where descending curve means higher than expected yield. RA-CUSUM, risk-adjusted cumulative sum.

In Hospital 1, median hospital stay before case 19 was 12 days (IQR: $9-12$ days) and after 9 days (IQR: 8-12 days), $\mathrm{P}=0.041$. In Hospital 2, stay before case 50 was 11 (IQR: 10-15) and after 7 (IQR: 7-9), $\mathrm{P}<0.001$.

\section{Survival}

Only two patients in Hospital 1 (1.5\%) and one in Hospital $2(1.8 \%)$ died during 90 -days after surgery. One-year mortality rate in respective hospitals were $7.3 \%$ and $18.4 \%$ 

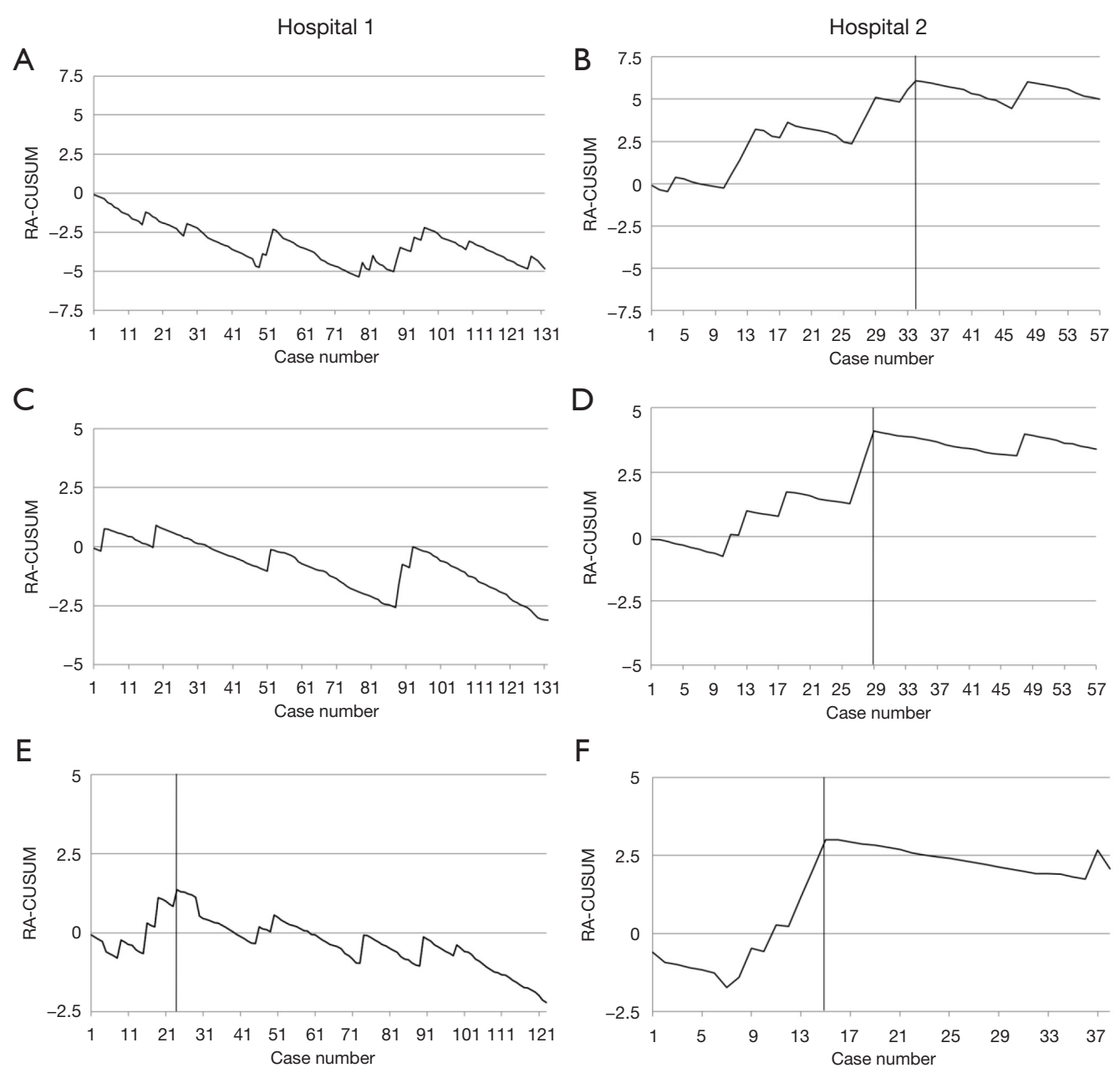

Figure 2 Learning curves presenting complications and mortality. RA-CUSUM of major complications (A,B), anastomosis leaks (C,D) and 1-year mortality (E,F). Hospital 1 is presented in the left, Hospital 2 in the right column. RA-CUSUM, risk-adjusted cumulative sum.

(Table 2). RA-CUSUM showed improvement in Hospital 1 after a plateau at 24 patients (Figure 2E). In Hospital 2, the improvement in performance occurred after 15 patients (Figure 2F).

In Hospital 1, 1-year mortality before case 24 was $16.7 \%$ and after 5.1\%, P=0.051. In Hospital 2, 1-year mortality before case 15 was $40 \%$ and after $4.3 \%, \mathrm{P}=0.068$.

\section{Comparison to Benchmark study}

Published Benchmark values are presented in Table 2. Despite the learning period in both Hospitals, all learningassociated parameters except lymph node yield were within suggested level in Hospital 1 (Table 2). Hospital 2 did not reach recommended benchmark values in total lymph node yield, R0-resection rate and 1-year mortality. Other parameters were within the suggested level including major complications, anastomosis leaks, re-admissions and 90-day mortality (Table 2).

\section{Discussion}

In this study, the learning curves for MIE in two programs varied significantly. The program started by an experienced MIE surgeon with a surgical team lacking prior MIE experience did not lead to extra morbidity. A proficient surgical team, both surgeons and other team members, for minimally invasive surrogate surgery and open esophagectomy did face a clear learning curve phase. The suggested benchmark (9) values regarding both primary outcome parameters, major complications and anastomotic leaks, were reached in both programs despite the learning phase. 
A major strength of this intent-to-treat study is the inclusion rate of $99 \%$ of patients eligible for curative-intent esophagectomy. Since the introduction of MIE, only two patients in these hospitals underwent open esophagectomy for cancer. The second strength is the completeness of the data. Since the patient data was acquired from prospective hospital registries and medical reports, relevant variables including comorbidities, stage and staging including the use of PET-CT, neoadjuvant treatment, and accurate description of complications were available, not often the case in registry-based studies. Both hospitals are the only centre performing esophagectomies and treating the complications in their regions. It is unlikely that any major complications were missed. Mortality data were complete, confirmed from the nationwide database with mandatory reporting. The third major strength is the outcome parameters. These differ significantly between studies focusing often on a single parameter. We included all relevant operation-related parameters in the CUSUM analyses. Our study has also limitations. With a small number of patients, definitive conclusions cannot be drawn. Especially in Hospital 2, the small cohort size can give false results regarding the true length of the learning curve. With a small cohort, it is possible that the learning curve has not been completed and future studies with an increased number of patients are needed. With these results recently published Benchmark outcomes were, however, mostly reached suggesting at least nearly completed learning phase. Previously, learning curve has mostly been examined, as in our study, using intraoperative variables and postoperative complications. Patient selection and proper staging are equally important in achieving high-quality outcomes. Furthermore, the hospital-specific cut-offs for operation time, blood loss, lymph node yield and length of stay make comparisons between the hospitals difficult, but take into account the different recording and assessment procedures between the hospitals. The aim was not to compare the hospitals, but to assess learning. In Hospital 2, despite guidelines (12), with PET-CT rate of only $45.7 \%$, the number of patients with undetected Stage IV disease was seemingly high. This should not impact short-term outcomes, but might explain the higher 1-year mortality in Hospital 2. Long-term survival assessment would have required a longer follow-up and needs further studies.

Previously, learning curve length has ranged from 35 to 175 cases based on the outcome parameter $(6,14,15)$. With the aimed leak rate set at $8 \%$, the length of learning curve in a recent multicentre study was 119 cases (4). So far, the largest study evaluating the length of learning curve in MIE is based on 14 European hospitals (5). In that study, this length with an anastomosis leak as an outcome parameter varied from 42 to 138 cases and with Textbook outcome (16) from 38 to 121 cases (5). Overall, the methodological heterogeneity between the learning curve studies makes comparisons difficult. In Hospital 2, downward decline was seen after 29 patients in the anastomosis leak rate, after 30 patients in operative time, and after 34 patients in the major complication rate. This is a consistent outcome and the reason for this relatively short learning curve could be the high surrogate surgery volume (17). Furthermore, in Hospital 2, learning curve effect was detectable in lymph node yield after 45 and hospital stay after 50 cases. A modest downward decline was evident already after 15 cases in operative blood loss. In blood loss, many studies have not detected any learning effect (6). Therefore, its role as a clinically significant outcome parameter in learning curve studies can be questioned.

In Hospital 1, an experienced MIE surgeon started the new program and the major complication rate and the anastomosis leak rate remained low and stable. Because no learning curve effect was evident in these primary outcomes, surgical expertise might have the biggest role in the learning curve phase of major surgery. Still, learning effect was observed in blood loss and lymph node yield. This might be more related to electrosurgery technology and image quality than the learning itself. The change in CUSUM for blood loss occurred simultaneously with the change of electrosurgery technique and camera system at around case 20 and 80 . Though during the whole study period en-bloc lymphadenectomy technique remained constant, a learning curve effect was detected. The decline in the slope of CUSUM plot for lymph node yield occurred just before the start of the study evaluating the ability of near-infrared imaging to detect lymphatic stations most likely containing the nodal metastases (13). In Hospital 2, median lymph node yield was suboptimal (median 17) compared to suggested Benchmark values (23 nodes) (9) but significant learning was observed with a median yield of 21 after 45 cases. Improved performance occurred later than in other measured parameters. Related to benchmark values, sample assessment practices in the pathology might affect the number of lymph nodes examined in the specimen (18).

Which factors influence the length of the learning curve? In a nationwide study, surgeons with a low operationspecific volume, but high rate of surrogate surgery, had similar in-hospital mortality rate after esophagectomy to 
high volume surgeons (17). Therefore, the outcomes from Hospital 2 suggests an important factor in the learning phase could be the volume of surrogate surgery. Higher centre MIE volumes have already been shown to shorten the learning curve phase (5). The outcome of Hospital 1 indicates the experience of the operating surgeon rather than the institutional learning curve phase is the major factor in the learning process. A short learning curve phase in Hospital 1 was, however, detected in the hospital stay. It seems logical that all members of the program need experience before the optimal outcome can be reached. It is surprising that visiting an expert clinic, completing a fellowship, or implementation of a new program under a proctor's supervision were not associated with more efficient learning in a recent multinational study (5). The safest way to start a new MIE program, though not often practical, could be the recruitment of an experienced MIE surgeon.

One-year mortality has not been an outcome parameter in previous learning curve studies of MIE. In robot-assisted laparoscopic hysterectomy for cervical cancer, learning curve phase is associated with survival outcomes (19). A peak in RA-CUSUM plot for 1-year mortality was observed in Hospital 1 at 24 cases and in Hospital 2 at 15 cases. A decline in one-year survival rate after uneventful minimally invasive $\mathrm{R} 0$-resection in quite unlikely caused by any operative factors. The cause is most likely related to patient selection and neoadjuvant therapy in Hospital 1. In Hospital 2, higher morbidity and patient selection during the early learning curve explains 1 -year outcomes.

It is noteworthy that although higher morbidity during the early learning curve occurred in Hospital 2, outcomes in both hospitals in the learning period were mostly superior compared to previously published Benchmark values (9). It seems, therefore, that adequate quality of surgery, according to these short-term outcomes, can be reached with a relatively low number of patients even in the learning period. Due to the fact that collaborating centres in the Benchmark study were often in the learning phase of their own, we have previously questioned whether the suggested Benchmarks are actually "best possible" (11). Therefore, optimal outcomes for MIE are yet to be determined. With a completed learning curve and excellent outcomes, the implication of MIE can be extended to difficult benign disorders such as end-stage achalasia, persisting strictures and poor functional outcomes after multiple antireflux operations with a low expected risk for major morbidity and mortality (20).

This study has several clinical implications. MIE seems to require a significant learning curve, though it may be shorter than previously suggested depending on surgeon's previous expertise. Therefore, an absolute requirement for MIE program is a good volume of surrogate surgery. On the other hand, surgeons with expertise in MIE can start de novo programs in hospitals with proficiency for other major cancer surgery and achieve excellent early outcomes. Therefore, the best way to reduce morbidity and to increase patient safety in the learning phase for MIE could be the recruitment of an expert as a team member.

\section{Conclusions}

This study indicates that the learning phase of an individual surgeon determines the outcomes of MIE, not the institutional volume or learning phase.

\section{Acknowledgments}

Funding: Instrumentarium Science Foundation $(\mathrm{OH})$, Mary and Georg C. Ehrnrooth Foundation $(\mathrm{OH})$ and Finnish State Research Funding $(\mathrm{OH}, \mathrm{HH})$, Finnish Cultural Foundation (HH), Vieno, Alli Suorsa's Healthcare Foundation (HH), The Finnish Cancer Foundation (JHK), Sigrid Juselius Foundation (JHK) and Päivikki and Sakari Sohlberg Foundation (JHK).

\section{Footnote}

Reporting Checklist: The authors have completed the STROBE reporting checklist. Available at https://dx.doi. org/10.21037/jtd-21-1063

Data Sharing Statement: Available at https://dx.doi. org/10.21037/jtd-21-1063

Peer Review File: Available at https://dx.doi.org/10.21037/ jtd-21-1063

Conflicts of Interest: All authors have completed the ICMJE uniform disclosure form (available at https://dx.doi. org/10.21037/jtd-21-1063). The authors have no conflicts of interest to declare.

Ethical Statement: The authors are accountable for all aspects of the work in ensuring that questions related to the accuracy or integrity of any part of the work are appropriately investigated and resolved. The study was 
conducted according to the guidelines of the Declaration of Helsinki (as revised in 2013). The study was approved by the Oulu University Hospital Ethics Committee and the hospital districts (committee's reference number 81/2008). The need to obtain informed consent from the study patients was waived by the Finnish National Authority for Medicolegal Affairs (VALVIRA).

Open Access Statement: This is an Open Access article distributed in accordance with the Creative Commons Attribution-NonCommercial-NoDerivs 4.0 International License (CC BY-NC-ND 4.0), which permits the noncommercial replication and distribution of the article with the strict proviso that no changes or edits are made and the original work is properly cited (including links to both the formal publication through the relevant DOI and the license). See: https://creativecommons.org/licenses/by-nc$\mathrm{nd} / 4.0 \%$.

\section{References}

1. Sung H, Ferlay J, Siegel RL, et al. Global Cancer Statistics 2020: GLOBOCAN Estimates of Incidence and Mortality Worldwide for 36 Cancers in 185 Countries. CA Cancer J Clin 2021;71:209-49.

2. Helminen O, Sihvo E, Gunn J, et al. Trends and results of oesophageal cancer surgery in Finland between 2004 and 2014. Eur J Cardiothorac Surg 2020;57:107-13.

3. Kauppila JH, Helminen O, Kytö V, et al. Short-Term Outcomes Following Minimally Invasive and Open Esophagectomy: A Population-Based Study from Finland and Sweden. Ann Surg Oncol 2018;25:326-32.

4. van Workum F, Stenstra MHBC, Berkelmans GHK, et al. Learning Curve and Associated Morbidity of Minimally Invasive Esophagectomy: A Retrospective Multicenter Study. Ann Surg 2019;269:88-94.

5. Claassen L, Hannink G, Luyer MDP, et al. Learning Curves of Ivor Lewis Totally Minimally Invasive Esophagectomy by Hospital and Surgeon Characteristics: A Retrospective Multi-National Cohort Study. Ann Surg 2021. [Epub ahead of print]. doi: 10.1097/ SLA.0000000000004801.

6. Claassen L, van Workum F, Rosman C. Learning curve and postoperative outcomes of minimally invasive esophagectomy. J Thorac Dis 2019;11:S777-85.

7. Dindo D, Demartines N, Clavien PA. Classification of surgical complications: a new proposal with evaluation in a cohort of 6336 patients and results of a survey. Ann Surg 2004;240:205-13.

8. Low DE, Alderson D, Cecconello I, et al. International Consensus on Standardization of Data Collection for Complications Associated With Esophagectomy: Esophagectomy Complications Consensus Group (ECCG). Ann Surg 2015;262:286-94.

9. Schmidt HM, Gisbertz SS, Moons J, et al. Defining Benchmarks for Transthoracic Esophagectomy: A Multicenter Analysis of Total Minimally Invasive Esophagectomy in Low Risk Patients. Ann Surg 2017;266:814-21.

10. Bolsin S, Colson M. The use of the Cusum technique in the assessment of trainee competence in new procedures. Int J Qual Health Care 2000;12:433-8.

11. Helminen O, Mrena J, Sihvo E. Benchmark values for transthoracic esophagectomy are not set as the defined "best possible"-a validation study. J Thorac Dis 2018;10:4085-93.

12. Lordick F, Mariette C, Haustermans K, et al. Oesophageal cancer: ESMO Clinical Practice Guidelines for diagnosis, treatment and follow-up. Ann Oncol 2016;27:v50-57.

13. Helminen O, Mrena J, Sihvo E. Near-infrared imageguided lymphatic mapping in minimally invasive oesophagectomy of distal oesophageal cancer. Eur J Cardiothorac Surg 2017;52:952-7.

14. Lin J, Kang M, Chen C, et al. Thoracolaparoscopy oesophagectomy and extensive two-field lymphadenectomy for oesophageal cancer: introduction and teaching of a new technique in a high-volume centre. Eur J Cardiothorac Surg 2013;43:115-21.

15. Tapias LF, Morse CR. Minimally invasive Ivor Lewis esophagectomy: description of a learning curve. J Am Coll Surg 2014;218:1130-40.

16. Busweiler LA, Schouwenburg MG, van Berge Henegouwen MI, et al. Textbook outcome as a composite measure in oesophagogastric cancer surgery. Br J Surg 2017;104:742-50.

17. Modrall JG, Minter RM, Minhajuddin A, et al. The Surgeon Volume-outcome Relationship: Not Yet Ready for Policy. Ann Surg 2018;267:863-7.

18. Pucher PH, Green M, Bateman AC, et al. Variation in histopathological assessment and association with surgical quality indicators following oesophagectomy. Br J Surg 2021;108:74-9.

19. Eoh KJ, Lee JY, Nam EJ, et al. The institutional 
learning curve is associated with survival outcomes of robotic radical hysterectomy for early-stage cervical cancer-a retrospective study. BMC Cancer 2020;20:152.

Cite this article as: Helminen O, Kauppila JH, Saviaro H, Yannopoulos F, Meriläinen S, Koivukangas V, Huhta H, Mrena J, Saarnio J, Sihvo E. Minimally invasive esophagectomy learning curves with different types of background experience. J Thorac Dis 2021;13(11):6261-6271. doi: 10.21037/jtd-21-1063
20. Guo W, Yang S, Li H. Esophagectomy with gastric conduit reconstruction for benign disease: extreme but important. Ann Transl Med 2018;6:117. 